\title{
Neuronal damage and shortening of lifespan in C.elegans by peritoneal dialysis fluid: Protection by glyoxalase-1
}

\author{
ANDREA SCHLOTTERER ${ }^{1 *}$, FRIEDERIKE PFISTERER $^{2 *}$, GEORGI KUKUDOV $^{1}$, \\ BRITTA HECKMANN ${ }^{3}$, DANIEL HENRIQUEZ ${ }^{4}$, CHRISTIAN MORATH $^{5}$, BERNHARD K. KRÄMER ${ }^{1}$, \\ HANS-PETER HAMMES ${ }^{1}$, VEDAT SCHWENGER ${ }^{6}$ and MICHAEL MORCOS ${ }^{1}$ \\ ${ }^{1}$ Fifth Medical Department, Medical Faculty Mannheim, Heidelberg University, D-68167 Mannheim; Departments of \\ ${ }^{2}$ Medicine I and Clinical Chemistry, and ${ }^{3}$ Dermatology, Heidelberg University, D-69120 Heidelberg, Germany; \\ ${ }^{4}$ Department of Nephrology, Centre Hospitalier du Nord, 9080 Ettelbruck, Luxembourg; ${ }^{5}$ Department of \\ Nephrology, Heidelberg University, D-69120 Heidelberg; ${ }^{6}$ Klinik für Nieren-, Hochdruck- und \\ Autoimmunerkrankungen, Klinikum Stuttgart, D-70174 Stuttgart, Germany
}

Received November 22, 2017; Accepted March 12, 2018

DOI: $10.3892 /$ br.2018.1085

\begin{abstract}
Glucose and glucose degradation products (GDPs), contained in peritoneal dialysis (PD) fluids, contribute to the formation of advanced glycation end-products (AGEs). Local damaging effects, resulting in functional impairment of the peritoneal membrane, are well studied. It is also supposed that detoxification of AGE precursors by glyoxalase-1 (GLO1) has beneficial effects on GDP-mediated toxicity. The aim of the current study was to analyze systemic detrimental effects of PD fluids and their prevention by glyoxlase-1. Wild-type and GLO1-overexpressing Caenorhabditis elegans (C.elegans) were cultivated in the presence of low- and high-GDP PD fluids containing 1.5 or $4 \%$ glucose. Lifespan, neuronal integrity and neuronal functions were subsequently studied. The higher concentrations of glucose and GDP content resulted in a decrease of maximum lifespan by $2(\mathrm{P}<0.01)$ and 9 days $(\mathrm{P}<0.001)$, respectively. Exposure to low- and high-GDP fluids caused reduction of neuronal integrity by $34(\mathrm{P}<0.05)$ and $41 \%$ $(\mathrm{P}<0.05)$. Cultivation of animals in the presence of low-GDP fluid containing $4 \%$ glucose caused significant impairment of neuronal function, reducing relative and absolute head motility by $58.5(\mathrm{P}<0.01)$ and $56.7 \%(\mathrm{P}<0.01)$, respectively; and relative and absolute tail motility by $55.1(\mathrm{P}<0.05)$ and $55.0 \%(\mathrm{P}<0.05)$, respectively. Taken together, GLO1 overexpression protected from glucose-induced lifespan reduction, neurostructural damage and neurofunctional damage under
\end{abstract}

Correspondence to: Dr Andrea Schlotterer, Fifth Medical Department, Medical Faculty Mannheim, Heidelberg University, Theodor-Kutzer-Ufer 1-3, D-68167 Mannheim, Germany

E-mail: andrea.schlotterer@medma.uni-heidelberg.de

*Contributed equally

Key words: glyoxalase I, peritoneal dialysis, neurons, life expectancy
low-GDP-conditions. In conclusion, both glucose and GDP content in PD fluids have systemic impact on the lifespan and neuronal integrity of $C$. elegans. Detoxification of reactive metabolites by GLO1 overexpression was sufficient to protect lifespan, neuronal integrity and neuronal function in a low-GDP environment. These data emphasize the relevance of the GLO1 detoxifying pathway as a potential therapeutic target in the treatment of reactive metabolite-mediated pathologies.

\section{Introduction}

Advanced glycation end-products (AGEs) are a diverse group of oxidant compounds with pathogenic significance in diabetes and in several other chronic diseases (for reviews see refs. 1,2). AGEs are generated by non-enzymatic reactions between reducing sugars and free amino groups of proteins, lipids and nucleic acids (3). Generation of AGEs has been mainly studied in the context of food preparation, and dietary intake of AGE forms a part of normal metabolism (4). However, when high levels of AGEs accumulate in tissues or the blood stream, they cause oxidative stress and promote inflammation by binding to cell surface receptors or cross-linking with body proteins, altering their structure and function (for review see ref. 5).

Peritoneal dialysis (PD) is applied in patients with end-stage kidney disease to increase preservation of residual renal function. Despite PD treatment, prevalence of cardiovascular events is considerably increased in those patients, and they are the leading cause of mortality (6). Furthermore, inflammation and the resulting cumulative peritoneal membrane injury contribute to the progression of chronic kidney disease $(7,8)$. Glucose degradation products (GDPs) in the PD fluids have been identified as the major initiators of inflammation and linked to the failure of ultrafiltration and degradation of the peritoneal membrane following PD (9).

Previous studies have identified 3-deoxyglucosone (3-DG), 3,4-dideoxyglucosone-3-ene (3,4-DGE) and methylglyoxal (MG) as the most bioreactive GDPs in PD fluids $(10,11)$. Acting as reactive carbonyl compounds, these metabolites 
cause protein modifications and ultimately the formation of AGEs (12), which contribute to functional impairment of human peritoneal mesothelial cells (HPMC) via AGE-AGE specific receptor (AGE-RAGE) interaction (13). Indeed, the use of low-GDP PD fluids decreased markers of inflammation: both local interleukin 6 levels in dialysates (14) and systemic serum C-reactive protein levels (15). While local damaging effects of GDPs on peritoneal mesothelial cells are well studied, little is known of the systemic effects (16).

Damaging effects of AGEs are physiologically counteracted with various salvage pathways, among which glyoxalase-1 (GLO1) is of outstanding importance. GLO1 detoxifies MG, thus reducing the formation of AGEs (17-19). Suppression of MG-mediated glycation by GLO1 is particularly important in diabetes and uremia, where the blood plasma concentration of MG is increased (20). Morcos et al (21) previously demonstrated that overexpression of glod-4, the Caenorhabditis elegans (C.elegans) ortholog of GLO1, decreased mitochondrial protein modification and extended lifespan. Therefore, C. elegans overexpressing glod-4 were used as a model organism to investigate the effect of the degradation product MG on AGE formation (21).

In the present study, the systemic effects of the glucose and GDP contents of PD fluids were analysed in the nematode C. elegans, by using lifespan, neuronal integrity and neuronal function as surrogate parameters for the damaging effects of reactive metabolites. C.elegans is a well-established model in the study of neurodegeneration as well as in aging research, benefitting from an accessible and characterized nervous system and a lifespan short enough to screen for ageing-related degenerative damages (22).

More specifically, the current study focused on MG, one of the major GDPs generated during heat sterilization of conventional fluids used for PD (23). Previous studies have demonstrated that increased GLO1 activity, acting as a detoxification system for MG, has beneficial effects on GDP-mediated toxicity in HPMCs (24). To the best of our knowledge, the present study is the first report using $C$. elegans with the aim of demonstrating the protective capability of the GLO1 detoxifying pathway against PD fluid-induced damages and of indicating its relevance as a potential therapeutic target.

\section{Materials and methods}

C. elegans maintenance. The wild-type strain $\mathrm{N} 2$ and the pan-neuronal green fluorescent protein (GFP)-expressing strain NW1229 were provided by the Caenorhabditis Genetics Center (College of Biological Sciences, Saint Paul, MN, USA), which is funded by the National Institutes of Health Office of Research Infrastructure Programs (program no. P40 OD010440). In NW1229, GFP is expressed as a rgef-1 (sequence name, F25B3.3) reporter fusion in post-mitotic neurons throughout the nervous system (pan-neuronally), beginning at the late comma stage of embryo development (25). The glod-4/GLO1 transgenic C.elegans (strain VH725, GLO1-Tg) was generated by our group and has been described previously (21).

Age-synchronized embryos were hatched, grown and cultivated throughout their lifespan on nematode growth medium (NGM) agar (A1296; Sigma-Aldrich; Merck KGaA, Darmstadt, Germany) in $60 \mathrm{~mm}$ Petri plates (25 animals per plate) and maintained at $20^{\circ} \mathrm{C}$ in a temperature-controlled incubator at ambient air humidity (75\%). As a food source, $100 \mu \mathrm{l}$ of a standardized Escherichia coli OP50 (Caenorhabditis Genetics Center, Saint Paul, MN, USA) overnight culture (optical density, 1.5) were added to the surface of each NGM plate.

PD fluid treatment. PD fluids contained either minimal concentrations of GDPs (low-GDP fluid, Gambrosol trio $10^{\circledR}$ double-chamber PD fluid; Baxter International, Inc., Deerfield, IL, USA) or conventional GDP concentrations (high-GDP fluid, Gambrosol ${ }^{\circledR}$ single-chamber PD fluid; Baxter International, Inc.). Low- and high-GDP fluids differed with respect to $\mathrm{pH}(6.6$ vs. 5.5) and concentrations of GDPs: 3-DG (12.3 vs. $118 \mu \mathrm{mol} / 1), 3,4-\mathrm{DGE}$ (below detection limit vs. $10 \mu \mathrm{mol} / \mathrm{l})$ and $\mathrm{MG}(<2.8 \mathrm{vs} .5 .3 \mu \mathrm{mol} / \mathrm{l})$, determined as described previously (26).

Glucose concentrations were adjusted to 1.5 or $4 \%$ glucose by addition of GDP-free glucose stock solution to obtain low-GDP PD fluid containing $1.5 \%$ (L-1.5) or $4 \%$ (L-4) glucose, or high-GDP PD fluid containing $1.5 \%(\mathrm{H}-1.5)$ or $4 \%(\mathrm{H}-4)$ glucose. The samples were sterile filtered $(0.02 \mu \mathrm{m}$; Sarstedt AG \& Co., Nümbrecht, Germany) and incubated without any preservatives at $37^{\circ} \mathrm{C}$ for 7 days to ensure GDP formation.

For PD fluid treatment, the food source was supplemented with $100 \mu \mathrm{l}$ of L-1.5, L-4, H-1.5 or H-4. Feeding procedures were repeated every $72 \mathrm{~h}$ for determination of lifespan or daily for evaluation of neuronal integrity and function. For control treatments, standard maintenance medium was used (M9 buffer, containing $22 \mathrm{mM} \mathrm{KH} \mathrm{PO}_{4}, 42 \mathrm{mM} \mathrm{Na}_{2} \mathrm{HPO}_{4}$, $86 \mathrm{mM} \mathrm{NaCl}$, adjusted to $\mathrm{pH} 7.0$, autoclaved and supplemented with $1 \mathrm{mM} \mathrm{MgSO}_{4}$; all reagents from Sigma-Aldrich; Merck KGaA).

Determination of lifespan. To evaluate effects on lifespan, Kaplan-Meier survival curves were calculated. To prevent progeny production, NGM plates containing $300 \mu \mathrm{g} / \mathrm{ml} 5$-fluorodesoxyuridine (F0503; Sigma-Aldrich; Merck KGaA) were used. For lifespan analysis, the pre-fertile period of adulthood was defined as $t=0$. Animals were regarded to have succumbed if they did not move following a repeated mechanical stimulus. Animals were excluded if they had crawled away from the plate or contained internally hatched larvae. Experiments were performed in triplicates with 50 animals each.

Evaluation of neuronal integrity and neuronal functions. Neuronal integrity and neuronal functions of $C$. elegans were used as accessible surrogate parameters to quantify the extent of glucose and GDP-induced damages (22). Neuronal integrity was assessed by quantifying fluorescence of the pan-neuronal GFP-expressing C. elegans (strain NW1229), as described previously (27-29). Briefly, GFP fluorescence of animals was recorded (BioTek FLx800) and quantified (Gen5 v1 software; both from BioTek Instruments, Inc., Winooski, VT, USA). Experiments were performed in triplicates. To determine the impact on neuronal function, animals were recorded (Moticam 1000; Beyersdörfer $\mathrm{GmbH}$, Mandelbachtal, Germany) and different parameters of animal motility were analyzed as described previously (30). In brief, a 
worm tracking software program (WormTracker 4.0; Thomas Bornhaupt, Neustadt adW., Germany) was used to split the recorded videos into single frames and to locate the head and tail positions of individual animals. These data were then used to calculate the head and tail velocities in relation to the plate surface (absolute) as well as in relation to the center of the body (relative), resulting in values for absolute head motility $(\mathrm{mm} / \mathrm{sec})$, relative head motility $(\mathrm{mm} / \mathrm{sec})$, absolute tail motility $(\mathrm{mm} / \mathrm{sec})$ and relative tail motility $(\mathrm{mm} / \mathrm{sec})$. In contrast to a simple description of whole nematode motility, these values are more specific parameters of anterior and posterior locomotor ability (31).

Statistical analysis. StatView 5.0 software (SAS Institute, Inc., Cary, NC, USA) was used for statistical analysis. Log-rank tests were used to analyze differences between Kaplan-Meier survival curves. Data on neuronal integrity and function are expressed as the mean \pm standard error of the mean. Student's t-tests were used to analyze differences between values of two groups. Differences with $\mathrm{P}<0.05$ were considered statistically significant.

\section{Results}

Lifespan is inversely affected by the glucose/GDP content of $P D$ fluids and GLO-1. To evaluate the effects of glucose and GDPs on the lifespan of C. elegans, four treatments groups were established: Low-GDP, low glucose (1.5\%); low-GDP, high glucose (4\%); high-GDP, low glucose; and high-GDP, high-glucose. In preliminary experiments in the current study, it was confirmed that low-GDP, low glucose PD fluid (L-1.5) did not significantly affect lifespan when compared with animals treated with standard maintenance medium (M9 buffer; P $>0.05$; data not shown). The higher concentration of GDPs significantly reduced the maximum lifespan of C. elegans, under both low-glucose (from 30 to 21 days, $\mathrm{P}<0.001$ ) and high-glucose conditions (from 28 to 19 days, $\mathrm{P}<0.001)$. Similarly, increased glucose reduced the maximum lifespan further, under both low-GDP (from 30 to 28 days; $\mathrm{P}<0.01$ ) and high-GDP conditions (from 21 to 19 days; $\mathrm{P}<0.05$; Fig. 1A).

To assess the effect of endogenous GLO1, animals overexpressing this enzyme were used, and again lifespan was measured following culture in high or low GDP, and high or low glucose concentrations. The higher concentrations of GDPs reduced the maximum lifespan of GLO1 transgenic C. elegans to a similar extent as observed in wild-type animals (from 27 to 21 days under low-glucose conditions, $\mathrm{P}<0.001$; and from 27 to 19 days under high-glucose conditions, $\mathrm{P}<0.001$ ). However, in the low-GDP group, overexpression of GLO1 abolished the effect of high glucose ( $\mathrm{P}>0.05$; Fig. 1B).

Reduction of neuronal integrity by PD fluids is prevented by GLO-1. To investigate the effect of glucose and GDP content on neuronal integrity, C.elegans with neuronal GFP expression were used. In this setting, quantification of GFP fluorescence allows assessment of neuronal integrity as described previously (29). In preliminary experiments in the current study, it was confirmed that standard M9 buffer did not significantly affect neuronal fluorescence over the study period of 7 days
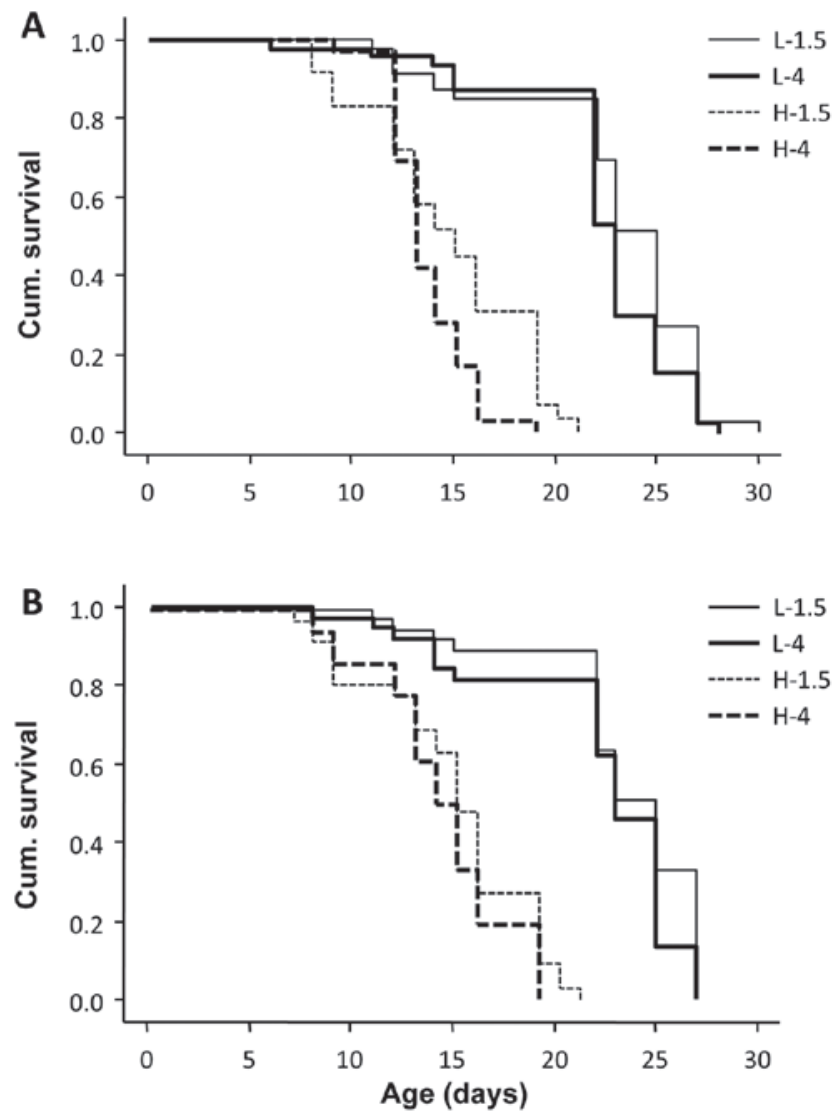

Figure 1.Effects of PD fluids and GLO-1 on lifespan. Kaplan-Meier graphs of the fraction of Caenorhabditis elegans alive show survival curves of (A) wild-type and (B) GLO-1-overexpressing animals, cultivated in the presence of low- GDP PD fluid containing 1.5 (L-1.5) or $4 \%$ glucose (L-4), or high-GDP PD fluid containing $1.5(\mathrm{H}-1.5)$ or $4 \%$ glucose $(\mathrm{H}-4)$. Results are shown for a representative experiment out of three independent experiments. PD, peritoneal dialysis; GLO-1, glyoxalase-1; GDP, glucose degradation product.

( $P>0.05$; data not shown). Exposure of wild-type animals to PD fluids led to a decrease in signal intensity of neuronal GFP at all GDP and glucose concentrations $(\mathrm{P}<0.05)$, indicative of neuronal damage. Fluorescence reduction ranged from $34 \%$ in low-GDP fluids to $41 \%$ in high-GDP fluids. The presence of additional glucose had no further effect (Control; Fig. 2A). GLO1 overexpression protected animals from neurostructural damage in the low-GDP group, but not in the high-GDP group (GLO1-Tg; Fig. 2A). Representative images of animals without neuronal damage and with reduced neuronal integrity are presented in Fig. 2B and C, respectively.

Neuronal function. To correlate PD fluid-induced defects in neuronal structure with impairment of neuronal functions, different parameters of animal motility were quantified. As GLO1 overexpression was indicated to prevent neuronal damages only under low-GDP conditions, these experiments were performed under low-GDP conditions and low or high glucose only.

Firstly, movement of the head, relative to the body (relative head motility) and to the surface (absolute head motility), was determined, since it is the most descriptive parameter for overall animal motility $(30,31)$. No significant changes were observed in the relative and absolute head motilities of a control group cultivated in GDP-free medium over a period 


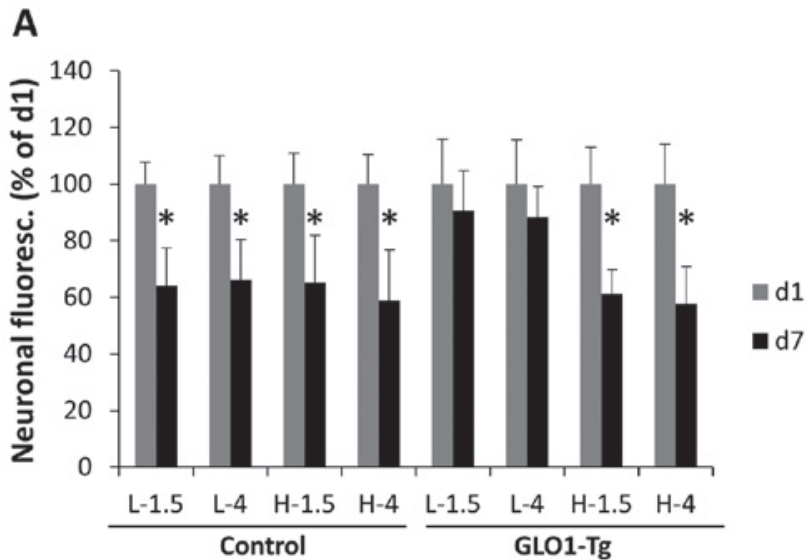

B

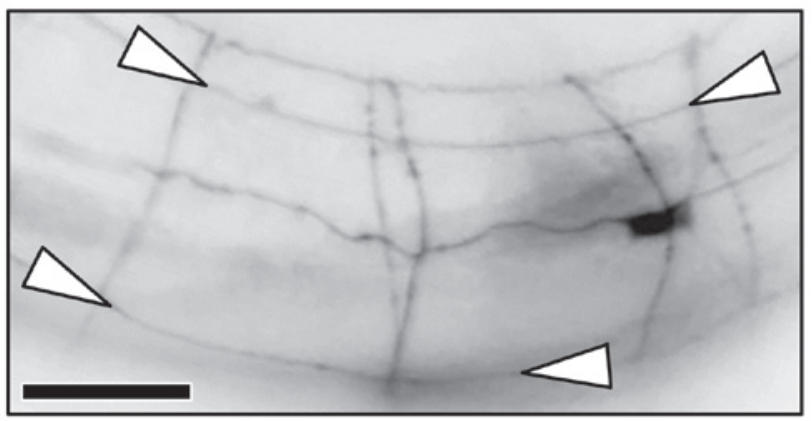

C

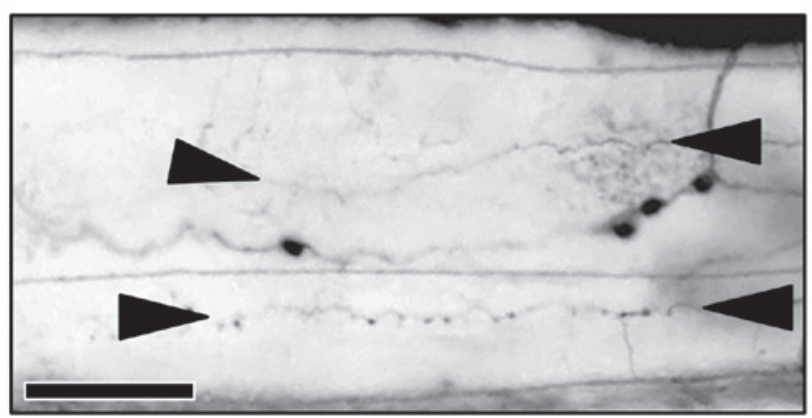

Figure 2. Neuronal integrity. (A) Neuronal integrity was assessed by fluorescence measurement of pan-neuronal green fluorescent protein-expressing Caenorhabditis elegans, either without (Control) or with GLO-1 transgenic overexpression (GLO1-Tg), exposed over a period of 1 day (d1, grey columns) or 7 days (d7, black columns) to low-GDP PD fluid containing $1.5 \%$ (L-1.5) or $4 \%$ glucose (L-4), or high-GDP PD fluid containing $1.5 \%(\mathrm{H}-1.5)$ or $4 \%$ glucose (H-4). Fluorescence was reduced as follows: by $36 \%$ (Control, L-1.5), 34\% (Control, L-4), 35\% (Control, H-1.5), 41\% (Control, H-4), 9\% (GLO1-Tg, L-1.5), 12\% (GLO1-Tg, L-4), 39\% (GLO1-Tg, H-1.5) and 42\% (GLO1-Tg, $\mathrm{H}-4)$. Results are the means with standard error of the mean of three independent experiments. ${ }^{*} \mathrm{P}<0.05$ for $\mathrm{d} 1 \mathrm{vs}$. $\mathrm{d} 7 \mathrm{under}$ each treatment condition (calculated using the unpaired Student's t-test). (B and C) Representative images of animals without neuronal damage and with reduced neuronal integrity. Shown are corresponding sections of the central body region from (B) animals with intact nerve cords (ends are marked with white arrowheads), and (C) animals with damaged nerve cords, indicated by beading-like lesions (ends are marked by black arrowheads). Scale bars, $0.03 \mathrm{~mm}$ (magnification, x50). PD, peritoneal dialysis; GLO-1, glyoxalase-1; GDP, glucose degradation product.

of 7 days (C-4, M9 buffer containing 4\% glucose) (Fig. 3A and B). Exposure of wild-type $C$. elegans to low-GDP fluids at $1.5 \%$ glucose (L-1.5) did not reduce relative and absolute head motilities significantly (Fig. 3A and B). When exposed to low-GDP fluids with $4 \%$ glucose (L-4), a significant decline in mean relative head motility, from 0.146 to $0.061 \mathrm{~mm} / \mathrm{sec}$
(58.5\%; P<0.01) was detected (Fig. 3A). Similarly, mean absolute head motility was decreased from 0.119 to $0.051 \mathrm{~mm} / \mathrm{sec}$ (56.7\%; P $<0.01$; Fig. 3B). Therefore, a rise in glucose content to $4 \%$ promoted GDP-induced neuronal damages to a degree not only affecting structural integrity, but also functional parameters. Similar to their influence on neuronal structure, glucose and GDPs appeared to contribute concertedly to the detrimental effects of PD fluids on neuronal function, since control groups treated with GDP-free medium at $4 \%$ glucose did not experience functional impairment.

GLO1 overexpression protected animals from glucose and GDP-induced neurofunctional damage (Fig. 3C and D). This confirmed the neuroprotective effect of GLO1 against damage induced by low-GDP PD fluids containing up to $4 \%$ glucose. Again, no significant changes were measured in the head motilities of the control groups (C-4, P>0.05; Fig. $3 \mathrm{C}$ and D).

Next, tail motility, both relative to the body (relative tail motility) and absolute to the surface (absolute tail motility), was determined. Low-GDP fluids with $1.5 \%$ glucose did not affect relative and absolute tail motilities over a period of 7 days (Fig. 3E and F). However, low-GDP fluid with $4 \%$ glucose significantly reduced the mean relative tail motility from 0.098 to $0.044 \mathrm{~mm} / \mathrm{sec}$ motility $(55.1 \%$; $\mathrm{P}<0.05$; Fig. $3 \mathrm{E})$, and mean absolute tail motility from 0.086 to $0.039 \mathrm{~mm} / \mathrm{sec}(55.0 \%$; $\mathrm{P}<0.05$; Fig. $3 \mathrm{~F}$ ). In the control group, following exposure to GDP-free medium with $4 \%$ glucose, no significant changes were observed in the relative and absolute tail motilities (Fig. 3E and F). The protective capacity of GLO1 overexpression against neurofunctional damage induced by low-GDP PD fluids containing up to $4 \%$ glucose was also confirmed by tail motility: motility at 1.5 and $4 \%$ glucose was not reduced significantly in these animals (Fig. $3 \mathrm{G}$ and $\mathrm{H}$ ).

Thus, a second neurofunctional parameter of tail motility appeared to be affected by GDP-induced damages, resulting from PD fluids containing an elevated glucose level of $4 \%$. Confirmed by the observations regarding neuronal structure and head motilities, both glucose and GDP content in PD fluids affected neuronal functions in C.elegans.

\section{Discussion}

PD fluids contain glucose at different concentrations, dependent on the intended ultrafiltration rate. Breakdown of glucose gives rise to the formation of glucose degradation products (GDPs), a mechanism accelerated during the heat sterilization process and particularly in a low-pH environment (32). Single-chamber PD fluids, undergoing heat sterilization in the presence of glucose, consequently contain high concentrations of GDPs. The high-GDP fluid used in the current study contained $5.3 \mu \mathrm{mol} / 1 \mathrm{MG}$ at a pH of 5.5 (26). PD fluids with high GDP content are considered to be less biocompatible, as indicated by their inhibitory effect on cell growth (10). In order to reduce GDP content, double-chamber PD fluids have been implemented where glucose is added only following the heat sterilization process to limit GDP formation (16). The low-GDP fluid used in the current study contained lower MG concentrations $(<2.8 \mu \mathrm{mol} / \mathrm{l})$ at a higher $\mathrm{pH}(6.6)(26)$. Additional approaches to decrease GDP levels of PD fluids, besides reduction of glucose content and adjustment of $\mathrm{pH}$, include the use of filter sterilization (33). 
A

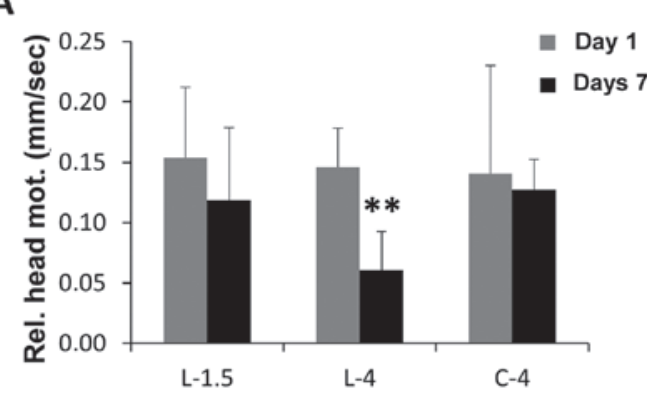

C

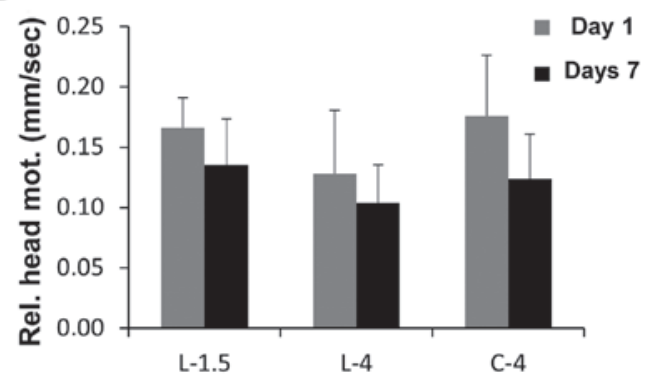

B

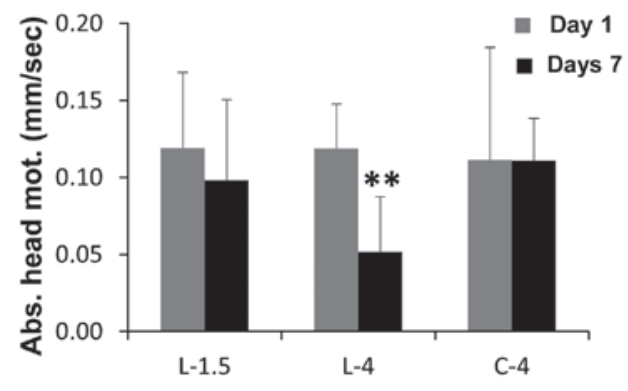

D

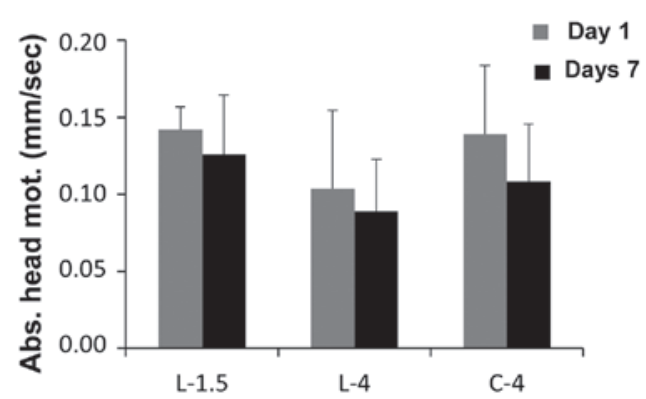

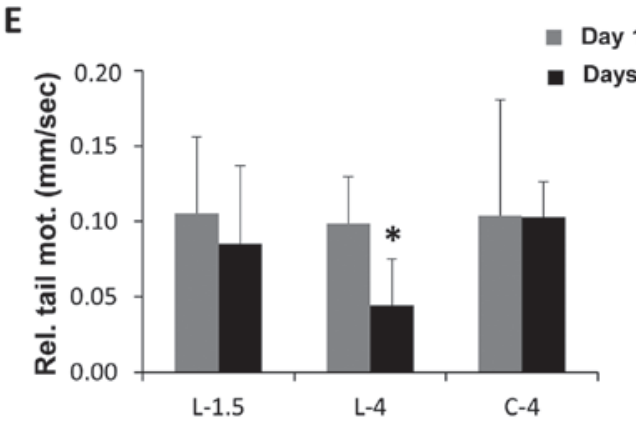

G

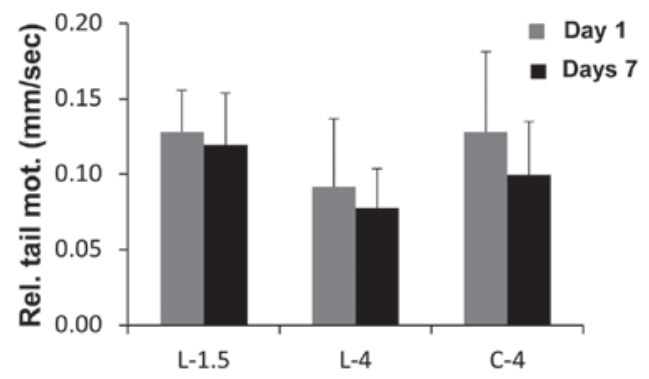

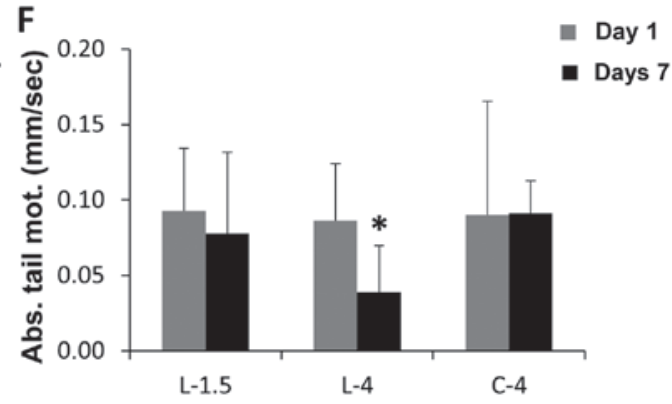

H

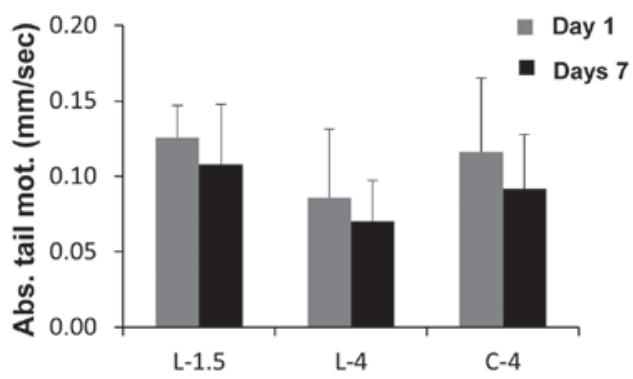

Figure 3. Neuronal function. (A-H) Neuronal function was measured in (A, B, E and F) wild-type and (C, D, G and H) GLO-1-overexpressing animals treated for 1 day (grey columns) or 7 days (black columns) with low-GDP PD fluid containing 1.5\% (L-1.5) or 4\% glucose (L-4), or GDP-free control medium (M9 buffer containing 4\% glucose, C-4), by quantification of (A and C) relative and (B and D) absolute head motility, and (E and $\mathrm{G})$ relative and $(\mathrm{F}$ and $\mathrm{H})$ absolute tail motility. Motility was reduced as follows: by $23.1 \%$ (A, L-1.5), 58.5\% (A, L-4), 9.1\% (A, C-4), 17.4\% (B, L-1.5), 56.7\% (B, L-4), 0.3\% (B, C-4), 18.3\% (C, L-1.5), $18.5 \%$ (C, L-4), 29.7\% (C, C-4), 11.3\% (D, L-1.5), 14.3\% (D, L-4), 22.1\% (D, C-4), 18.9\% (E, L-1.5), 55.1\% (E, L-4), 0.9\% (E, C-4), 16.3\% (F, L-1.5), 55.0\% (F, L-4), -1.0\% (F, C-4), 6.7\% (G, L-1.5), 15.3\% (G, L-4), 22.1\% (G, C-4), 13.9\% (H, L-1.5), 18.0\% (H, L-4), and 21.1\% (H, C-4). Results are shown as mean velocities with standard error of the mean of three independent experiments, each with 10 animals per group. ${ }^{*} \mathrm{P}<0.05$ and ${ }^{* *} \mathrm{P}<0.01$ for $\mathrm{d} 1 \mathrm{vs}$. $\mathrm{d} 7 \mathrm{under}$ each treatment condition (calculated using the unpaired Student's t-test). PD, peritoneal dialysis; GLO-1, glyoxalase-1; GDP, glucose degradation product.

3-DG, 3,4-DGE and MG have been previously identified as the most bioreactive GDPs formed in PD fluids (10,11), and lead to formation of AGEs, which indirectly mediates GDP toxicity via RAGE activation $(13,34)$. GDP toxicity correlates with functional and morphological changes in peritoneal membrane integrity, thus degrading ultrafiltration quality and ultimately limiting use of the peritoneal membrane as a dialysis substrate membrane (33).
Previous studies have not only illustrated that GDPs affect the peritoneal membrane, but also depicted the consequences on systemic circulation. It is apparent that systemic circulation of GDPs promotes chronic kidney disease, which is mediated via AGE-RAGE interaction (35). In vivo studies have indicated resorption of GDPs from the peritoneal cavity, accompanied by an increase in plasma AGE compounds (26). Rapid GDP adsorption was confirmed in a later study describing the 
association of exposure to high- and low-GDP fluids with plasma AGE levels (16). GDP-dependent systemic increase of AGE formation has been causally linked with inflammation, neoangiogenesis and fibrosis via AGE-RAGE interaction, since RAGE-deficient animals are protected (13).

The aim of the current study was to evaluate the systemic effect of GDP toxicity, beyond its direct impact on the peritoneal membrane. For this purpose the study used $C$. elegans, a widely acknowledged model organism used for investigation of the effects of toxic metabolites (36), such as AGEs formed in the presence of reactive $\alpha$-oxoaldehydes, mainly carboxymethyllysine (CML), carboxyethyllysine (CEL) and imidazolones (21), as well as in toxicity studies of the effects of pharmaceutically used drugs, such as immunosuppressants (37), on lifespan and neuronal function (38-43). Despite the lack of a distinct renal system, basic pathophysiological processes observed in C. elegans may also be applicable to humans, since this animal features a $70 \%$ homology to human genes $(37,44)$.

Previous studies have demonstrated neurotoxic effects of AGEs (45) and oxidative stress (46). Morcos et al (21) demonstrated in C. elegans that AGE-induced mitochondrial modifications increased levels of oxidative stress. The accumulation of AGEs, including CML and CEL, accounted for modification of various key proteins, thus inducing age-related damage on a molecular level.

In the current study, it was demonstrated in C. elegans that reduction of lifespan and neuronal integrity was not only caused by increased levels of glucose but also by elevated GDP content in PD fluids. Therefore, both glucose and GDPs contained in PD fluids may contribute to AGE formation and accelerate aging via RAGE-mediated pathways.

Notably, protection of lifespan and neuronal integrity by GLO1 overexpression was restricted to a low-GDP environment. This suggests that detoxification of MG by GLO1 is not sufficient to prevent the deleterious effects of PD fluids containing high levels of various GDPs. The failure of GLO1 overexpression to exert significant beneficial effects in a high-GDP environment may also be due to the fact that GLO1 activity is dependent on the presence of glutathione as a co-factor (47). Previous studies have demonstrated that the GLO/glutathione system in human peritoneal mesothelial cells is impaired by conventional high-GDP fluids; a protective effect could only be established following replenishment of glutathione (24). Thus, substitution of the glutathione precursor L-2-oxothiazolidine-4-carboxylic acid may prevent the loss of GLO1 activity under high-GDP exposure.

Impairment of neuronal function appears to occur secondary to neurostructural damage. During the exposure time of 7 days, neuronal integrity was affected by PD fluids at $1.5 \%$ glucose, while parameters of animal motility were reduced only by PD fluids at $4 \%$ glucose. GLO1 overexpression protected various parameters of neuronal function, in line with the observed beneficial effects on neuronal integrity. Upon exposure to low-GDP PD fluids at $4 \%$ glucose, the parameters of relative head motility, absolute head motility, relative tail motility and absolute tail motility were rescued in GLO1-Tg animals.

Overall, the current study demonstrated the significant impact of both glucose and GDP content in PD fluids on lifespan, neuronal integrity and neuronal function in $C$. elegans. The protective effects induced by GLO1 overexpression emphasize the relevance of the GLO1 detoxifying pathway as a potential therapeutic target in the treatment of reactive metabolite-mediated pathologies. Further studies into the vascular system of higher organisms are needed to translate these findings into clinical practice.

\section{Acknowledgements}

Not applicable.

\section{Funding}

The current study was supported by grants from the German Research Foundation (Collaborative Research Center 1118).

\section{Availability of data and materials}

All data generated or analyzed during this study are included in this published article.

\section{Authors' contributions}

AS made substantial contributions to analysis and interpretation of data and was involved in drafting the manuscript. FP, $\mathrm{GK}$ and $\mathrm{BH}$ made substantial contributions to acquisition of data and were involved in drafting the manuscript. $\mathrm{DH}, \mathrm{CM}$, BKK, HPH, VS and MM made substantial contributions to conception and design and were involved in revising the manuscript critically for important intellectual content. All authors read and approved the final manuscript.

\section{Ethics approval and consent to participate}

Not applicable.

\section{Consent for publication}

Not applicable.

\section{Competing interests}

The authors declare that they have no competing interests.

\section{References}

1. Davis KE, Prasad C, Vijayagopal P, Juma S and Imrhan V: Advanced glycation end products, inflammation, and chronic metabolic diseases: Links in a chain? Crit Rev Food Sci Nutr 56: 989-998, 2016.

2. Vlassara $\mathrm{H}$ and Uribarri J: Advanced glycation end products (AGE) and diabetes: Cause, effect, or both? Curr Diab Rep 14: 453, 2014.

3. Hellwig $M$ and Henle T: Baking, ageing, diabetes: A short history of the Maillard reaction. Angew Chem Int Ed Engl 53: 10316-10329, 2014

4. Uribarri J, del Castillo MD, de la Maza MP, Filip R, Gugliucci A, Luevano-Contreras C, Macías-Cervantes MH, Markowicz Bastos DH, Medrano A, Menini T, et al: Dietary advanced glycation end products and their role in health and disease. Adv Nutr 6: 461-473, 2015.

5. Ott C, Jacobs K, Haucke E, Navarrete Santos A, Grune T and Simm A: Role of advanced glycation end products in cellular signaling. Redox Biol 2: 411-429, 2014. 
6. Johnson DW, Dent H, Hawley CM, McDonald SP, Rosman JB, Brown FG, Bannister K and Wiggins KJ: Association of dialysis modality and cardiovascular mortality in incident dialysis patients. Clin J Am Soc Nephrol 4: 1620-1628, 2009.

7. Borazan A, Ustün H, Ustundag Y, Aydemir S, Bayraktaroglu T, Sert $\mathrm{M}$ and Yilmaz A: The effects of peritoneal dialysis and hemodialysis on serum tumor necrosis factor-alpha, interleukin-6, interleukin-10 and C-reactive-protein levels. Mediators Inflamm 13: 201-204, 2004

8. Chung SH, Heimbürger O, Stenvinkel P, Bergström J and Lindholm B: Association between inflammation and changes in residual renal function and peritoneal transport rate during the first year of dialysis. Nephrol Dial Transplant 16: 2240-2245, 2001.

9. Jörres A, Witowski J and Bender TO: PD and loss of peritoneal function. Prilozi 28: 275-281, 2007.

10. Erixon M, Wieslander A, Lindén T, Carlsson O, Forsbäck G Svensson E, Jönsson JA and Kjellstrand P: How to avoid glucose degradation products in peritoneal dialysis fluids. Perit Dial Int 26: 490-497, 2006

11. Linden T, Forsback G, Deppisch R, Henle T and Wieslander A 3-Deoxyglucosone, a promoter of advanced glycation end products in fluids for peritoneal dialysis. Perit Dial Int 18: 290-293, 1998.

12. Miyata T, Horie K, Ueda Y, Fujita Y, Izuhara Y, Hirano H, Uchida K, Saito A, van Ypersele de Strihou C and Kurokawa K: Advanced glycation and lipidoxidation of the peritoneal membrane: Respective roles of serum and peritoneal fluid reactive carbonyl compounds. Kidney Int 58: 425-435, 2000.

13. Schwenger V, Morath C, Salava A, Amann K, Seregin Y, Deppisch R, Ritz E, Bierhaus A, Nawroth PP and Zeier M: Damage to the peritoneal membrane by glucose degradation products is mediated by the receptor for advanced glycation end-products J Am Soc Nephrol 17: 199-207, 2006.

14. Cooker LA, Luneburg P, Holmes CJ, Jones S, Topley N and Bicarbonate/Lactate Study Group: Interleukin-6 levels decrease in effluent from patients dialyzed with bicarbonate/lactate-based peritoneal dialysis solutions. Perit Dial Int 21 (Suppl 3): S102-107, 2001.

15. Szeto CC, Chow KM, Lam CW, Leung CB, Kwan BC, Chung KY, Law MC and Li PK: Clinical biocompatibility of a neutral peritoneal dialysis solution with minimal glucose-degradation products - a 1-year randomized control trial. Nephrol Dial Transplant 22: 552-559, 2007.

16. Schmitt CP, von Heyl D, Rieger S, Arbeiter K, Bonzel KE, Fischbach M, Misselwitz J, Pieper AK and Schaefer F; Mid European Pediatric Peritoneal Dialysis Study Group (MEPPS) Reduced systemic advanced glycation end products in children receiving peritoneal dialysis with low glucose degradation product content. Nephrol Dial Transplant 22: 2038-2044, 2007.

17. Thornalley PJ: Dietary AGEs and ALEs and risk to human health by their interaction with the receptor for advanced glycation endproducts (RAGE) - an introduction. Mol Nutr Food Res 51: 1107-1110, 2007

18. Thornalley PJ: Endogenous alpha-oxoaldehydes and formation of protein and nucleotide advanced glycation endproducts in tissue damage. Novartis Found Symp 285: 229-243; discussion 243-246, 2007.

19. Thornalley PJ: Glyoxalase I - structure, function and a critical role in the enzymatic defence against glycation. Biochem Soc Trans 31: 1343-1348, 2003

20. Thornalley PJ: Protein and nucleotide damage by glyoxal and methylglyoxal in physiological systems - role in ageing and disease. Drug Metabol Drug Interact 23: 125-150, 2008.

21. Morcos M, Du X, Pfisterer F, Hutter H, Sayed AA, Thornalley P, Ahmed N, Baynes J, Thorpe S, Kukudov G, et al: Glyoxalase-1 prevents mitochondrial protein modification and enhances lifespan in Caenorhabditis elegans. Aging Cell 7: 260-269, 2008

22. Mendler M, Schlotterer A, Morcos M and Nawroth PP: Understanding diabetic polyneuropathy and longevity: What can we learn from the nematode Caenorhabditis elegans? Exp Clin Endocrinol Diabetes 120: 182-183, 2012.

23. Schalkwijk CG, Posthuma N, ten Brink HJ, ter Wee PM and Teerlink T: Induction of 1,2-dicarbonyl compounds, intermediates in the formation of advanced glycation end-products, during heat-sterilization of glucose-based peritoneal dialysis fluids. Perit Dial Int 19: 325-333, 1999.

24. Korybalska K, Wisniewska-Elnur J, Trómińska J, Jörres A, Breborowicz A and Witowski J: The role of the glyoxalase pathway in reducing mesothelial toxicity of glucose degradation products. Perit Dial Int 26: 259-265, 2006.
25. Altun-Gultekin Z, Andachi Y, Tsalik EL,Pilgrim D, Kohara Y and Hobert O: A regulatory cascade of three homeobox genes, ceh-10, ttx-3 and ceh-23, controls cell fate specification of a defined interneuron class in C. elegans. Development 128: 1951-1969, 2001.

26. Zeier M, Schwenger V, Deppisch R, Haug U, Weigel K, Bahner U, Wanner C, Schneider H, Henle T and Ritz E: Glucose degradation products in PD fluids: Do they disappear from the peritoneal cavity and enter the systemic circulation? Kidney Int 63: 298-305, 2003.

27. Hutter H: Axonale Wegfindung im ventralen Nervenstrang von C. elegans. Tätigkeitsbericht, 2003 (In German)

28. Hobert O and Loria P: Uses of GFP in Caenorhabditis elegans. In: Green Fluorescent Protein: Properties, Applications, and Protocols. Chalfie M and Kain SR (eds). Vol 47. 2nd edition. John Wiley \& Sons, Inc., Hoboken, NJ, pp203-226, 2006.

29. Negga R, Rudd DA, Davis NS, Justice AN, Hatfield HE, Valente AL, Fields AS and Fitsanakis VA: Exposure to $\mathrm{Mn} / \mathrm{Zn}$ ethylene-bis-dithiocarbamate and glyphosate pesticides leads to neurodegeneration in Caenorhabditis elegans. Neurotoxicology 32 331-341, 2011

30. Mendler M, Schlotterer A, Ibrahim Y, Kukudov G, Fleming T, Bierhaus A, Riedinger C, Schwenger V, Herzig S, Hecker M, et al: daf-16/FOXO and glod-4/glyoxalase-1 are required for the life-prolonging effect of human insulin under high glucose conditions in Caenorhabditis elegans. Diabetologia 58: 393-401, 2015.

31. Schlotterer A, Greten HJ, Remppis BA, Kukudov G, Efferth T, Machado J, Humpert P, Hammes HP and Morcos M: Neuroprotection and antioxidative effects of Sijunzi Tang Decoction in the nematode Caenorhabditis elegans. Eur J Integr Med 8: 526-532, 2016.

32. Ortiz A, Wieslander A, Linden T, Santamaria B, Sanz A, Justo P, Sanchez-Niño MD, Benito A and Kjellstrand P: 3,4-DGE is important for side effects in peritoneal dialysis what about its role in diabetes. Curr Med Chem 13: 2695-2702, 2006

33. Choi HY, Kim DK, Lee TH, Moon SJ, Han SH, Lee JE, Kim BS, Park HC, Choi KH, Ha SK, et al: The clinical usefulness of peritoneal dialysis fluids with neutral $\mathrm{pH}$ and low glucose degradation product concentration: An open randomized prospective trial. Perit Dial Int 28: 174-182, 2008.

34. Schwenger V: GDP and AGE receptors: Mechanisms of peritoneal damage. Contrib Nephrol 150: 77-83, 2006.

35. Müller-Krebs S, Kihm LP, Zeier B, Gross ML, Deppisch R, Wieslander A, Henle T, Penndorf I, Oh J, Reiser J, et al: Renal toxicity mediated by glucose degradation products in a rat model of advanced renal failure. Eur J Clin Invest 38: 296-305, 2008.

36. Dengg M and van Meel JC: Caenorhabditis elegans as model system for rapid toxicity assessment of pharmaceutical compounds. J Pharmacol Toxicol Methods 50: 209-214, 2004.

37. Artal-Sanz M, de Jong L and Tavernarakis N: Caenorhabditis elegans: A versatile platform for drug discovery. Biotechnol J 1: 1405-1418, 2006

38. Ailion M, Inoue T, Weaver CI, Holdcraft RW and Thomas JH: Neurosecretory control of aging in Caenorhabditis elegans. Proc Natl Acad Sci USA 96: 7394-7397, 1999.

39. Boulianne GL: Neuronal regulation of lifespan: Clues from flies and worms. Mech Ageing Dev 122: 883-894, 2001

40. Geanacopoulos M: The determinants of lifespan in the nematode Caenorhabditis elegans: A short primer. Sci Prog 87: 227-247, 2004.

41. Luo Y: Long-lived worms and aging. Redox Rep 9: 65-69, 2004.

42. Paik YK, Jeong SK, Lee EY, Jeong PY and Shim YH C. elegans: An invaluable model organism for the proteomics studies of the cholesterol-mediated signaling pathway. Expert Rev Proteomics 3: 439-453, 2006.

43. Shen LL, Wang Y and Wang DY: Involvement of genes required for synaptic function in aging control in C. elegans. Neurosci Bull 23: 21-29, 2007.

44. Murakami S: Caenorhabditis elegans as a model system to study aging of learning and memory. Mol Neurobiol 35: 85-94, 2007.

45. Choei H, Sasaki N, Takeuchi M, Yoshida T, Ukai W, Yamagishi S, Kikuchi S and Saito T: Glyceraldehyde-derived advanced glycation end products in Alzheimer's disease. Acta Neuropathol 108: 189-193, 2004.

46. Fatokun AA, Stone TW and Smith RA: Oxidative stress in neurodegeneration and available means of protection. Front Biosci 13: 3288-3311, 2008.

47. Deponte M: Glutathione catalysis and the reaction mechanisms of glutathione-dependent enzymes. Biochim Biophys Acta 1830: 3217-3266, 2013. 